UDC: $517.518 .18: 517.965$

45 (LXXI) No.2 (2021), 127-142

DOI: $10.37560 /$ matbil21452129k

\title{
SOME NEW ESTIMATES USING GENERALIZED QUANTUM MONTGOMERY IDENTITY VIA STRONGLY PREINVEX FUNCTIONS OF HIGHER ORDER
}

\author{
ARTION KASHURI ${ }^{1}$, EZE R. NWAEZE ${ }^{2}$, AND ROZANA LIKO ${ }^{1}$
}

\begin{abstract}
In this paper, we obtain a new generalized version of the quantum Montgomery identity. Using this identity, some new estimates, for the class of strongly preinvex functions of higher order, are established. Furthermore, novel inequalities are deduced from our main results as special cases and in addition, recapture some known results as well. We anticipate that results presented herein will trigger further interest in this direction.
\end{abstract}

\section{INTRODUCTION}

Quantum calculus or q-calculus has received much attention in the last years and is served as bridge between mathematics and physics. Recently, Tariboon et al. in [18, defined q-derivative and q-integral as follows:

Definition 1.1. Let $\Psi:\left[e_{1}, e_{2}\right] \rightarrow \mathbb{R}$ be a continuous function and let $x \in\left[e_{1}, e_{2}\right]$ and $0<\mathrm{q}<1$ be a constant. Then the q-derivative on $\left[e_{1}, e_{2}\right]$ of function $\Psi(x)$ is defined as

$$
e_{1} D_{\mathrm{q}} \Psi(x)=\frac{\Psi(x)-\Psi\left(q x+(1-\mathrm{q}) e_{1}\right)}{(1-\mathrm{q})\left(x-e_{1}\right)}, x \neq e_{1} .
$$

We say that $\Psi(x)$ is q-differentiable on $\left[e_{1}, e_{2}\right]$ provided ${ }_{e_{1}} D_{\mathrm{q}} \Psi(x)$ exists for all $x \in\left[e_{1}, e_{2}\right]$.

Definition 1.2. Let $\Psi:\left[e_{1}, e_{2}\right] \subset \mathbb{R} \rightarrow \mathbb{R}$ be a continuous function. Then qintegral on $\left[e_{1}, e_{2}\right]$ is defined as

$$
\int_{e_{1}}^{x} \Psi(\ell)_{e_{1}} d_{\mathrm{q}} \ell=(1-\mathrm{q})\left(x-e_{1}\right) \sum_{n=0}^{\infty} \mathrm{q}^{n} \Psi\left(\mathrm{q}^{n} x+\left(1-\mathrm{q}^{n}\right) e_{1}\right)
$$

2010 Mathematics Subject Classification. Primary: 26A51 Secondary: 26A33, 26D07, 26D10, 26D15.

Key words and phrases. Montgomery identity, q-differentiable, strongly preinvex functions, Hölder inequality, power mean inequality. 
for $x \in\left[e_{1}, e_{2}\right]$.

For more details on q-calculus and certain q-analogues of classical inequalities, see $[1,,[3]-[5],[7],[10]-[13,,[17]-[21,,[23]$.

The following famous identity given in [[9]], is called Montgomery identity:

$$
\begin{gathered}
\Psi(x)=\frac{1}{e_{2}-e_{1}} \int_{e_{1}}^{e_{2}} \Psi(\ell) d \ell+\frac{1}{e_{2}-e_{1}} \int_{e_{1}}^{x}\left(\ell-e_{1}\right) \Psi^{\prime}(\ell) d \ell \\
+\frac{1}{e_{2}-e_{1}} \int_{x}^{e_{2}}\left(\ell-e_{2}\right) \Psi^{\prime}(\ell) d \ell,
\end{gathered}
$$

where the function $\Psi(x)$ is continuous on $\left[e_{1}, e_{2}\right]$ with a continuous first derivative in $\left(e_{1}, e_{2}\right)$.

By changing variable, the Montgomery identity 1.3 could be expressed as follows:

$$
\Psi(x)-\frac{1}{e_{2}-e_{1}} \int_{e_{1}}^{e_{2}} \Psi(\ell) d \ell=\left(e_{2}-e_{1}\right) \int_{0}^{1} H(\ell) \Psi^{\prime}\left((1-\ell) e_{1}+\ell e_{2}\right) d \ell
$$

where

$$
H(\ell):= \begin{cases}\ell, & \ell \in\left[0, \frac{x-e_{1}}{e_{2}-e_{1}}\right] \\ \ell-1, & \ell \in\left(\frac{x-e_{1}}{e_{2}-e_{1}}, 1\right] .\end{cases}
$$

We recall now some basic definitions for our study as follows:

Let $\mathbf{E}$ be a non-empty set, $\Psi: \mathbf{E} \rightarrow \mathbb{R}$ be a continuous functions and $\zeta: \mathbf{E} \times \mathbf{E} \rightarrow \mathbb{R}$ be a continuous bifunction.

Definition 1.3. 4] $A$ set $\mathbf{E} \subset \mathbb{R}$ is said to be invex with respect to bifunction $\zeta(.,)$, if

$$
e_{1}+\ell \zeta\left(e_{2}, e_{1}\right) \in \mathbf{E}, \quad \forall e_{1}, e_{2} \in \mathbf{E}, \ell \in[0,1] .
$$

Definition 1.4. 22] A function $\Psi: \mathbf{E} \rightarrow \mathbb{R}$ is said to be preinvex with respect to bifunction $\zeta(.,$.$) , if$

$$
\Psi\left(e_{1}+\ell \zeta\left(e_{2}, e_{1}\right)\right) \leq(1-\ell) \Psi\left(e_{1}\right)+\ell \Psi\left(e_{2}\right), \quad \forall e_{1}, e_{2} \in \mathbf{E}, \ell \in[0,1] .
$$

The notion of strongly convex functions was introduced by Karamardian [6] and Polyak [15].

Definition 1.5. A function $\Psi: \mathbf{E} \subseteq \mathbb{R} \longrightarrow \mathbb{R}$ is said to be strongly convex with modulus $\mu>0$, if

$$
\Psi\left((1-\ell) e_{1}+\ell e_{2}\right) \leq(1-\ell) \Psi\left(e_{1}\right)+\ell \Psi\left(e_{2}\right)-\mu \ell(1-\ell)\left(e_{2}-e_{1}\right)^{2}
$$

for all $e_{1}, e_{2} \in \mathbf{E}$ and $\ell \in[0,1]$. 
In [6], Karamardian noticed that every strongly monotone has a gradient map if and only if all differentiable function is strongly convex. Higher order strongly convex functions introduced by Lin et al. in [8], to abridge the research of linear programming with equilibrium constraints.

Definition 1.6. A function $\Psi: \mathbf{E} \subseteq \mathbb{R} \longrightarrow \mathbb{R}$ is said to be strongly convex with modulus $\mu>0$ and order $\sigma>0$, if

$$
\Psi\left((1-\ell) e_{1}+\ell e_{2}\right) \leq(1-\ell) \Psi\left(e_{1}\right)+\ell \Psi\left(e_{2}\right)-\mu \ell(1-\ell)\left(e_{2}-e_{1}\right)^{\sigma}
$$

for all $e_{1}, e_{2} \in \mathbf{E}$ and $\ell \in[0,1]$.

Recently, Awan et al. in [2], defined the following class of strongly preinvex functions of higher order.

Definition 1.7. A function $\Psi: \mathbf{E} \longrightarrow \mathbb{R}$ is said to be strongly preinvex with modulus $\mu>0$, order $\sigma>0$ and with respect to $\zeta: \mathbf{E} \times \mathbf{E} \longrightarrow \mathbb{R}$, if

$$
\Psi\left(e_{1}+\ell \zeta\left(e_{2}, e_{1}\right)\right) \leq(1-\ell) \Psi\left(e_{1}\right)+\ell \Psi\left(e_{2}\right)-\mu \ell(1-\ell) \zeta^{\sigma}\left(e_{2}, e_{1}\right)
$$

for all $e_{1}, e_{2} \in \mathbf{E}$ and $\ell \in[0,1]$.

Motivated by the above literatures, the main objective of this article is to obtain a generalization of the Montgomery identity given in 1.4 using the concepts of q-calculus. From this identity, several new and known q-analogues of integral inequalities involving strongly preinvex functions of higher order will be obtain. We also will discuss some new special cases of the main results. Finally, a brief conclusion will be provided.

\section{MAIN RESUlts}

Throughout this section, we shall let $P=\left[e_{1}, e_{1}+\zeta\left(e_{2}, e_{1}\right)\right]$ and $P^{\circ}$ denote the interior of $P$. For the sake of brevity, we define the following function $\wp_{\zeta}: P \rightarrow$ $[0,1]$ by

$$
\wp_{\zeta}(x):=\frac{x-e_{1}}{\zeta\left(e_{2}, e_{1}\right)}, \text { where } \zeta\left(e_{2}, e_{1}\right)>0, \quad \text { and } \quad x \in P .
$$

Lemma 1 (Generalized quantum Montgomery identity). If $\Psi: P \rightarrow \mathbb{R}$ is a q-differentiable function such that ${ }_{e} D_{\mathrm{q}} \Psi$ is quantum integrable on $P^{\circ}$, then the following identity holds:

$$
\begin{aligned}
\Psi(x) & -\frac{1}{\zeta\left(e_{2}, e_{1}\right)} \int_{e_{1}}^{e_{1}+\zeta\left(e_{2}, e_{1}\right)} \Psi(\ell)_{e_{1}} d_{\mathrm{q}} \ell=\zeta\left(e_{2}, e_{1}\right) \\
& \times \int_{0}^{1} T_{\mathrm{q}}(\ell)_{e_{1}} D_{\mathrm{q}} \Psi\left(e_{1}+\ell \zeta\left(e_{2}, e_{1}\right)\right)_{0} d_{\mathrm{q}} \ell
\end{aligned}
$$

where

$$
T_{\mathrm{q}}(\ell):=\left\{\begin{aligned}
\mathrm{q} \ell, & \ell \in\left[0, \wp_{\zeta}(x)\right] ; \\
\mathrm{q} \ell-1, & \ell \in\left(\wp_{\zeta}(x), 1\right] .
\end{aligned}\right.
$$


Proof. By using Definitions 1.1 and 1.2 we have

$$
\begin{aligned}
& \zeta\left(e_{2}, e_{1}\right) \int_{0}^{1} T_{\mathrm{q}}(\ell)_{e_{1}} D_{\mathrm{q}} \Psi\left(e_{1}+\ell \zeta\left(e_{2}, e_{1}\right)\right)_{0} d_{\mathrm{q}} \ell \\
& =\zeta\left(e_{2}, e_{1}\right)\left[\int_{0}^{\wp_{\zeta}(x)} \mathrm{q} \ell_{e_{1}} D_{\mathrm{q}} \Psi\left(e_{1}+\ell \zeta\left(e_{2}, e_{1}\right)\right)_{0} d_{\mathrm{q}} \ell+\right. \\
& \left.+\int_{\wp_{\zeta}(x)}^{1}(\mathrm{q} \ell-1)_{e_{1}} D_{\mathrm{q}} \Psi\left(e_{1}+\ell \zeta\left(e_{2}, e_{1}\right)\right)_{0} d_{\mathrm{q}} \ell\right] \\
& =\zeta\left(e_{2}, e_{1}\right)\left[\int_{0}^{\wp_{\zeta}(x)} \mathrm{q} \ell_{e_{1}} D_{\mathrm{q}} \Psi\left(e_{1}+\ell \zeta\left(e_{2}, e_{1}\right)\right)_{0} d_{\mathrm{q}} \ell+\right. \\
& \left.\int_{0}^{1}(\mathrm{q} \ell-1)_{e_{1}} D_{\mathrm{q}} \Psi\left(e_{1}+\ell \zeta\left(e_{2}, e_{1}\right)\right)_{0} d_{\mathrm{q}} \ell-\int_{0}^{\wp_{\zeta}(x)}(\mathrm{q} \ell-1)_{e_{1}} D_{\mathrm{q}} \Psi\left(e_{1}+\ell \zeta\left(e_{2}, e_{1}\right)\right)_{0} d_{\mathrm{q}} \ell\right] \\
& =\zeta\left(e_{2}, e_{1}\right)\left[\int_{0}^{1}(\mathrm{q} \ell-1) e_{e_{1}} D_{\mathrm{q}} \Psi\left(e_{1}+\ell \zeta\left(e_{2}, e_{1}\right)\right)_{0} d_{\mathrm{q}} \ell+\int_{0}^{\wp_{\zeta}(x)} e_{1} D_{\mathrm{q}} \Psi\left(e_{1}+\ell \zeta\left(e_{2}, e_{1}\right)\right)_{0} d_{\mathrm{q}} \ell\right] \\
& =\zeta\left(e_{2}, e_{1}\right)\left[\int_{0}^{1} \mathrm{q} \ell_{e_{1}} D_{\mathrm{q}} \Psi\left(e_{1}+\ell \zeta\left(e_{2}, e_{1}\right)\right)_{0} d_{\mathrm{q}} \ell-\int_{0}^{1} e_{1} D_{\mathrm{q}} \Psi\left(e_{1}+\ell \zeta\left(e_{2}, e_{1}\right)\right)_{0} d_{\mathrm{q}} \ell\right. \\
& \left.+\int_{0}^{\wp_{\zeta}(x)} e_{1} D_{\mathrm{q}} \Psi\left(e_{1}+\ell \zeta\left(e_{2}, e_{1}\right)\right)_{0} d_{\mathrm{q}} \ell\right] \\
& =\frac{1}{1-\mathrm{q}}\left[\begin{array}{c}
\mathrm{q}(1-\mathrm{q})\left[\begin{array}{c}
\sum_{n=0}^{\infty} \mathrm{q}^{n} \Psi\left(e_{1}+\mathrm{q}^{n} \zeta\left(e_{2}, e_{1}\right)\right) \\
-\sum_{n=0}^{\infty} \mathrm{q}^{n} \Psi\left(e_{1}+\mathrm{q}^{n+1} \zeta\left(e_{2}, e_{1}\right)\right)
\end{array}\right] \\
-(1-\mathrm{q})\left[\begin{array}{c}
\sum_{n=0}^{\infty} \mathrm{q}^{n} \frac{\Psi\left(e_{1}+\mathrm{q}^{n} \zeta\left(e_{2}, e_{1}\right)\right)}{\mathrm{q}^{n}} \\
-\sum_{n=0}^{\infty} \mathrm{q}^{n} \frac{\Psi\left(e_{1}+\mathrm{q}^{n+1} \zeta\left(e_{2}, e_{1}\right)\right)}{\mathrm{q}^{n}}
\end{array}\right] \\
+(1-\mathrm{q}) \wp_{\zeta}(x)\left[\begin{array}{c}
\sum_{n=0}^{\infty} \mathrm{q}^{n} \frac{\Psi\left(e_{1}+\mathrm{q}^{n} \wp_{\zeta}(x) \zeta\left(e_{2}, e_{1}\right)\right)}{\mathrm{q}^{n} \wp_{\zeta}(x)} \\
-\sum_{n=0}^{\infty} \mathrm{q}^{n} \frac{\Psi\left(e_{1}+\mathrm{q}^{n+1} \wp_{\zeta}(x) \zeta\left(e_{2}, e_{1}\right)\right)}{\mathrm{q}^{n} \wp_{\zeta}(x)}
\end{array}\right]
\end{array}\right]
\end{aligned}
$$




$$
\begin{aligned}
& {\left[\begin{array}{c}
\mathrm{q}\left[\begin{array}{c}
\sum_{n=0}^{\infty} \mathrm{q}^{n} \Psi\left(e_{1}+\mathrm{q}^{n} \zeta\left(e_{2}, e_{1}\right)\right) \\
-\sum_{n=0}^{\infty} \mathrm{q}^{n} \Psi\left(e_{1}+\mathrm{q}^{n+1} \zeta\left(e_{2}, e_{1}\right)\right)
\end{array}\right] \\
-\left[\begin{array}{c}
\sum_{n=0}^{\infty} \Psi\left(e_{1}+\mathrm{q}^{n} \zeta\left(e_{2}, e_{1}\right)\right) \\
-\sum_{n=0}^{\infty} \Psi\left(e_{1}+\mathrm{q}^{n+1} \zeta\left(e_{2}, e_{1}\right)\right)
\end{array}\right] \\
+\left[\begin{array}{c}
\sum_{n=0}^{\infty} \Psi\left(e_{1}+\mathrm{q}^{n} \wp_{\zeta}(x) \zeta\left(e_{2}, e_{1}\right)\right) \\
-\sum_{n=0}^{\infty} \Psi\left(e_{1}+\mathrm{q}^{n+1} \wp_{\zeta}(x) \zeta\left(e_{2}, e_{1}\right)\right)
\end{array}\right]
\end{array}\right]} \\
& {\left[\begin{array}{c}
\mathrm{q}\left[\begin{array}{c}
\sum_{n=0}^{\infty} \mathrm{q}^{n} \Psi\left(e_{1}+\mathrm{q}^{n} \zeta\left(e_{2}, e_{1}\right)\right) \\
-\frac{1}{\mathrm{q}} \sum_{n=1}^{\infty} \mathrm{q}^{n} \Psi\left(e_{1}+\mathrm{q}^{n} \zeta\left(e_{2}, e_{1}\right)\right)
\end{array}\right] \\
-\left[\begin{array}{c}
\sum_{n=0}^{\infty} \Psi\left(e_{1}+\mathrm{q}^{n} \zeta\left(e_{2}, e_{1}\right)\right) \\
-\sum_{n=1}^{\infty} \Psi\left(e_{1}+\mathrm{q}^{n} \zeta\left(e_{2}, e_{1}\right)\right)
\end{array}\right] \\
+\left[\begin{array}{c}
\sum_{n=0}^{\infty} \Psi\left(e_{1}+\mathrm{q}^{n} \wp_{\zeta}(x) \zeta\left(e_{2}, e_{1}\right)\right) \\
-\sum_{n=1}^{\infty} \Psi\left(e_{1}+\mathrm{q}^{n} \wp_{\zeta}(x) \zeta\left(e_{2}, e_{1}\right)\right)
\end{array}\right]
\end{array}\right]} \\
& =\left[\begin{array}{l}
\mathrm{q}\left[\left(1-\frac{1}{\mathrm{q}}\right) \sum_{n=0}^{\infty} \mathrm{q}^{n} \Psi\left(e_{1}+\mathrm{q}^{n} \zeta\left(e_{2}, e_{1}\right)\right)+\frac{\Psi\left(e_{1}+\zeta\left(e_{2}, e_{1}\right)\right)}{\mathrm{q}}\right] \\
-\Psi\left(e_{1}+\zeta\left(e_{2}, e_{1}\right)\right)+\Psi\left(e_{1}+\wp_{\zeta}(x) \zeta\left(e_{2}, e_{1}\right)\right)
\end{array}\right] \\
& =\Psi(x)-(1-\mathrm{q}) \sum_{n=0}^{\infty} \mathrm{q}^{n} \Psi\left(e_{1}+\mathrm{q}^{n} \zeta\left(e_{2}, e_{1}\right)\right) \\
& =\Psi(x)-\frac{1}{\zeta\left(e_{2}, e_{1}\right)} \int_{e_{1}}^{e_{1}+\zeta\left(e_{2}, e_{1}\right)} \Psi(\ell)_{e_{1}} d_{\mathrm{q}} \ell .
\end{aligned}
$$

The proof of our lemma is completed.

Remark 2.1: Taking $q \rightarrow 1^{-}$in Lemma 1, we have

$$
\begin{array}{r}
\Psi(x)-\frac{1}{\zeta\left(e_{2}, e_{1}\right)} \int_{e_{1}}^{e_{1}+\zeta\left(e_{2}, e_{1}\right)} \Psi(\ell) d \ell=\zeta\left(e_{2}, e_{1}\right) \\
\times\left[\int_{0}^{\wp_{\zeta}(x)} \ell \Psi^{\prime}\left(e_{1}+\ell \zeta\left(e_{2}, e_{1}\right)\right) d \ell+\int_{{ }_{\zeta}(x)}^{1}(\ell-1) \Psi^{\prime}\left(e_{1}+\ell \zeta\left(e_{2}, e_{1}\right)\right) d \ell\right] .
\end{array}
$$

Remark 2.2: Taking $\zeta\left(e_{2}, e_{1}\right)=e_{2}-e_{1}$ in Lemma 1, we get [7, Lemma 3]. 
Remark 2.3: Taking $q \rightarrow 1^{-}$and $\zeta\left(e_{2}, e_{1}\right)=e_{2}-e_{1}$ in Lemma 1 , we obtain the Montgomery identity given in 1.4.

Remark 2.4: Taking $x=\frac{2 e_{1}+\zeta\left(e_{2}, e_{1}\right)}{2}$ in Remark 2.1, we get [14, Lemma 3.10].

$$
\begin{gathered}
\Psi\left(\frac{2 e_{1}+\zeta\left(e_{2}, e_{1}\right)}{2}\right)-\frac{1}{\zeta\left(e_{2}, e_{1}\right)} \int_{e_{1}}^{e_{1}+\zeta\left(e_{2}, e_{1}\right)} \Psi(\ell) d \ell=\zeta\left(e_{2}, e_{1}\right) \\
\times\left[\int_{0}^{\frac{1}{2}} \ell \Psi^{\prime}\left(e_{1}+\ell \zeta\left(e_{2}, e_{1}\right)\right) d \ell+\int_{\frac{1}{2}}^{1}(\ell-1) \Psi^{\prime}\left(e_{1}+\ell \zeta\left(e_{2}, e_{1}\right)\right) d \ell\right] .
\end{gathered}
$$

Remark 2.5: Taking $x=\frac{q e_{1}+e_{2}}{1+q}$ and $\zeta\left(e_{2}, e_{1}\right)=e_{2}-e_{1}$ in Lemma 1 , we obtain equality (4.1) of [1].

$$
\begin{aligned}
& \Psi\left(\frac{q e_{1}+e_{2}}{1+\mathrm{q}}\right)-\frac{1}{e_{2}-e_{1}} \int_{e_{1}}^{e_{2}} \Psi(\ell)_{e_{1}} d_{\mathrm{q}} \ell \\
& =\left(e_{2}-e_{1}\right)\left[\begin{array}{c}
\int_{0}^{\frac{1}{1+q}} \mathrm{q} \ell_{e_{1}} D_{\mathrm{q}} \Psi\left((1-\ell) e_{1}+\ell e_{2}\right)_{0} d_{\mathrm{q}} \ell \\
+\int_{\frac{1}{1+q}}^{1}(\mathrm{q} \ell-1)_{e_{1}} D_{\mathrm{q}} \Psi\left((1-\ell) e_{1}+\ell e_{2}\right)_{0} d_{\mathrm{q}} \ell
\end{array}\right] .
\end{aligned}
$$

Remark 2.6: Taking $x=\frac{e_{1}+\mathrm{q}\left(e_{1}+\zeta\left(e_{2}, e_{1}\right)\right)}{1+\mathrm{q}}$ in Lemma 1 , we have

$$
\begin{aligned}
& \Psi\left(\frac{e_{1}+\mathrm{q}\left(e_{1}+\zeta\left(e_{2}, e_{1}\right)\right)}{1+\mathrm{q}}\right)-\frac{1}{\zeta\left(e_{2}, e_{1}\right)} \int_{e_{1}}^{e_{1}+\zeta\left(e_{2}, e_{1}\right)} \Psi(\ell)_{e_{1}} d_{\mathrm{q}} \ell \\
& =\zeta\left(e_{2}, e_{1}\right)\left[\begin{array}{c}
\int_{0}^{\frac{\mathrm{q}}{1+q}} \mathrm{q} \ell_{e_{1}} D_{\mathrm{q}} \Psi\left(e_{1}+\ell \zeta\left(e_{2}, e_{1}\right)\right)_{0} d_{\mathrm{q}} \ell \\
+\int_{\frac{\mathrm{q}}{1+\mathrm{q}}}^{1}(\mathrm{q} \ell-1)_{e_{1}} D_{\mathrm{q}} \Psi\left(e_{1}+\ell \zeta\left(e_{2}, e_{1}\right)\right)_{0} d_{\mathrm{q}} \ell
\end{array}\right] .
\end{aligned}
$$

Now, using Lemma 1, we can derive our main results for the class of higher order strongly preinvex functions.

Theorem 1. Let $\Psi: P \rightarrow \mathbb{R}$ be a function such that ${ }_{e_{1}} D_{\mathrm{q}} \Psi$ is q-integrable on $P^{\circ}$. If $\left.\left.\right|_{e_{1}} D_{\mathrm{q}} \Psi\right|^{r}$ is strongly preinvex functions of order $\sigma>0$ with modulus $\mu>0$ on $P$, then for $r>1$ and $p^{-1}+r^{-1}=1$, the following inequality holds:

$$
\left|\Psi(x)-\frac{1}{\zeta\left(e_{2}, e_{1}\right)} \int_{e_{1}}^{e_{1}+\zeta\left(e_{2}, e_{1}\right)} \Psi(\ell)_{e_{1}} d_{\mathrm{q}} \ell\right| \leq \mathrm{q} \zeta\left(e_{2}, e_{1}\right)
$$




$$
\times\left[\begin{array}{c}
{\left[L_{1}\left(\mathrm{q}, e_{1}, e_{2}, x\right)\right]^{\frac{1}{p}}\left[\begin{array}{c}
\left|e_{1} D_{\mathrm{q}} \Psi\left(e_{1}\right)\right|^{r} L_{2}\left(\mathrm{q}, e_{1}, e_{2}, x\right) \\
+\left|e_{1} D_{\mathrm{q}} \Psi\left(e_{2}\right)\right|^{r} L_{3}\left(\mathrm{q}, e_{1}, e_{2}, x\right)- \\
-\mu \zeta^{\sigma}\left(e_{2}, e_{1}\right) P_{1}\left(\mathrm{q}, e_{1}, e_{2}, x\right)
\end{array}\right]^{\frac{1}{r}}} \\
+\left[L_{4}\left(\mathrm{q}, e_{1}, e_{2}, x\right)\right]^{\frac{1}{p}}\left[\begin{array}{c}
\left|e_{1} D_{\mathrm{q}} \Psi\left(e_{1}\right)\right|^{r} L_{5}\left(\mathrm{q}, e_{1}, e_{2}, x\right) \\
+\left|e_{1} D_{\mathrm{q}} \Psi\left(e_{2}\right)\right|^{r} L_{6}\left(\mathrm{q}, e_{1}, e_{2}, x\right)- \\
-\mu \zeta^{\sigma}\left(e_{2}, e_{1}\right) P_{2}\left(\mathrm{q}, e_{1}, e_{2}, x\right)
\end{array}\right]^{\frac{1}{r}}
\end{array}\right],
$$

where

$$
\begin{aligned}
& L_{1}\left(\mathrm{q}, e_{1}, e_{2}, x\right):=\int_{0}^{\wp_{\zeta}(x)} \ell^{p}{ }_{0} d_{\mathrm{q}} \ell=\left[\wp_{\zeta}(x)\right]^{p} \frac{(1-\mathrm{q})}{1-\mathrm{q}^{p+1}}, \\
& L_{2}\left(\mathrm{q}, e_{1}, e_{2}, x\right):=\int_{0}^{\wp_{\zeta}(x)}(1-\ell)_{0} d_{\mathrm{q}} \ell=\wp_{\zeta}(x)-\frac{1}{1+\mathrm{q}}\left[\wp_{\zeta}(x)\right]^{2} \text {, } \\
& L_{3}\left(\mathrm{q}, e_{1}, e_{2}, x\right):=\int_{0}^{\wp_{\zeta}(x)} \ell_{0} d_{\mathrm{q}} \ell=\frac{1}{1+\mathrm{q}}\left[\wp_{\zeta}(x)\right]^{2}, \\
& L_{4}\left(\mathrm{q}, e_{1}, e_{2}, x\right):=\int_{\wp_{\zeta}(x)}^{1}\left(\ell-\frac{1}{\mathrm{q}}\right)^{p}{ }_{0} d_{\mathrm{q}} \ell=(1-\mathrm{q})\left[\begin{array}{c}
\sum_{n=0}^{\infty} \mathrm{q}^{n}\left(\mathrm{q}^{n}-\frac{1}{\mathrm{q}}\right)^{p}- \\
\wp_{\zeta}(x) \sum_{n=0}^{\infty} \mathrm{q}^{n}\left(\mathrm{q}^{n} \wp_{\zeta}(x)-\frac{1}{\mathrm{q}}\right)^{p}
\end{array}\right], \\
& L_{5}\left(\mathrm{q}, e_{1}, e_{2}, x\right):=\int_{\wp_{\zeta}(x)}^{1}(1-\ell){ }_{0} d_{\mathrm{q}} \ell=\frac{\mathrm{q}}{1+\mathrm{q}}-\wp_{\zeta}(x)+\frac{1}{1+\mathrm{q}}\left[\wp_{\zeta}(x)\right]^{2} \text {, } \\
& L_{6}\left(\mathrm{q}, e_{1}, e_{2}, x\right):=\int_{\wp_{\zeta}(x)}^{1} \ell_{0} d_{\mathrm{q}} \ell=\frac{1}{1+\mathrm{q}}\left(1-\left[\wp_{\zeta}(x)\right]^{2}\right) \text {, }
\end{aligned}
$$

and

$$
\begin{aligned}
& P_{1}\left(\mathrm{q}, e_{1}, e_{2}, x\right):=\int_{0}^{\wp_{\zeta}(x)} \ell(1-\ell)_{0} d_{\mathrm{q}} \ell=\frac{1}{1+\mathrm{q}}\left[\wp_{\zeta}(x)\right]^{2}-\frac{1}{1+\mathrm{q}+\mathrm{q}^{2}}\left[\wp_{\zeta}(x)\right]^{3}, \\
& P_{2}\left(\mathrm{q}, e_{1}, e_{2}, x\right):=\int_{\wp_{\zeta}(x)}^{1} \ell(1-\ell)_{0} d_{\mathrm{q}} \ell=L_{6}\left(\mathrm{q}, e_{1}, e_{2}, x\right)-\frac{1}{1+\mathrm{q}+\mathrm{q}^{2}}\left(1-\left[\wp_{\zeta}(x)\right]^{3}\right) .
\end{aligned}
$$

Proof. Using Lemma 1] strongly preinvexity of order $\sigma>0$ with modulus $\mu>0$ of $\left|e_{1} D_{\mathrm{q}} \Psi\right|^{r}$ and Hölder's inequality, we get

$$
\left|\Psi(x)-\frac{1}{\zeta\left(e_{2}, e_{1}\right)} \int_{e_{1}}^{e_{1}+\zeta\left(e_{2}, e_{1}\right)} \Psi(\ell)_{e_{1}} d_{\mathrm{q}} \ell\right|
$$


134

A. KASHURI, E. R. NWAEZE, AND R. LIKO

$$
\begin{aligned}
& \leq \zeta\left(e_{2}, e_{1}\right)\left[\begin{array}{c}
\left.\left.\int_{0}^{\wp_{\zeta}(x)} \mathrm{q} \ell\right|_{e_{1}} D_{\mathrm{q}} \Psi\left(e_{1}+\ell \zeta\left(e_{2}, e_{1}\right)\right)\right|_{0} d_{\mathrm{q}} \ell \\
+\left.\left.\int_{\wp_{\zeta}(x)}^{1}(\mathrm{q} \ell-1)\right|_{e_{1}} D_{\mathrm{q}} \Psi\left(e_{1}+\ell \zeta\left(e_{2}, e_{1}\right)\right)\right|_{0} d_{\mathrm{q}} \ell
\end{array}\right] \\
& \leq \zeta\left(e_{2}, e_{1}\right)\left[\left(\int_{0}^{\wp_{\zeta}(x)}(\mathrm{q} \ell)^{p}{ }_{0} d_{\mathrm{q}} \ell\right)^{\frac{1}{p}}\left(\int_{0}^{\wp_{\zeta}(x)}\left|e_{1} D_{\mathrm{q}} \Psi\left(e_{1}+\ell \zeta\left(e_{2}, e_{1}\right)\right)\right|^{r}{ }_{0} d_{\mathrm{q}} \ell\right)^{\frac{1}{r}}\right. \\
& \left.+\left(\int_{\rho_{\zeta}(x)}^{1}(\mathrm{q} \ell-1)^{p}{ }_{0} d_{\mathrm{q}} \ell\right)^{\frac{1}{p}}\left(\int_{\rho_{\zeta}(x)}^{1}\left|e_{e_{1}} D_{\mathrm{q}} \Psi\left(e_{1}+\ell \zeta\left(e_{2}, e_{1}\right)\right)\right|^{r}{ }_{0} d_{\mathrm{q}} \ell\right)^{\frac{1}{r}}\right] \\
& \leq \mathrm{q} \zeta\left(e_{2}, e_{1}\right)\left\{\left(\int_{0}^{\wp_{\zeta}(x)} \ell^{p}{ }_{0} d_{\mathrm{q}} \ell\right)^{\frac{1}{p}}\right. \\
& \times\left(\left|e_{e_{1}} D_{\mathrm{q}} \Psi\left(e_{1}\right)\right|^{r} \int_{0}^{\wp_{\zeta}(x)}(1-\ell)_{0} d_{\mathrm{q}} \ell+\left.\left.\right|_{e_{1}} D_{\mathrm{q}} \Psi\left(e_{2}\right)\right|^{r} \int_{0}^{\wp_{\zeta}(x)} \ell_{0} d_{\mathrm{q}} \ell-\right. \\
& \left.-\mu \zeta^{\sigma}\left(e_{2}, e_{1}\right) \int_{0}^{\wp_{\zeta}(x)} \ell(1-\ell)_{0} d_{\mathrm{q}} \ell\right)^{\frac{1}{r}}+\left(\int_{\wp_{\zeta}(x)}^{1}\left(\ell-\frac{1}{\mathrm{q}}\right)^{p}{ }_{0} d_{\mathrm{q}} \ell\right)^{\frac{1}{p}} \\
& \times\left(\left|e_{1} D_{\mathrm{q}} \Psi\left(e_{1}\right)\right|^{r} \int_{\wp_{\zeta}(x)}^{1}(1-\ell)_{0} d_{\mathrm{q}} \ell+\right. \\
& \left.\left.+\left|e_{1} D_{\mathrm{q}} \Psi\left(e_{2}\right)\right|^{r} \int_{\wp \zeta(x)}^{1} \ell_{0} d_{\mathrm{q}} \ell-\mu \zeta^{\sigma}\left(e_{2}, e_{1}\right) \int_{\wp \zeta(x)}^{1} \ell(1-\ell)_{0} d_{\mathrm{q}} \ell\right)^{\frac{1}{r}}\right\} .
\end{aligned}
$$

The proof of Theorem 1 is completed.

We point out some special cases of Theorem 1.

Corollary 1.1. I. Taking $\mathrm{q} \rightarrow 1^{-}$in Theorem 1, we have

$$
\left|\Psi(x)-\frac{1}{\zeta\left(e_{2}, e_{1}\right)} \int_{e_{1}}^{e_{1}+\zeta\left(e_{2}, e_{1}\right)} \Psi(\ell) d \ell\right| \leq \zeta\left(e_{2}, e_{1}\right)
$$


NEW ESTIMATES USING GENERALIZED QUANTUM MONTGOMERY IDENTITY 135

$$
\left[\begin{array}{c}
{\left[L_{7}\left(e_{1}, e_{2}, x\right)\right]^{\frac{1}{p}}\left[\begin{array}{c}
\left|\Psi^{\prime}\left(e_{1}\right)\right|^{r} L_{8}\left(e_{1}, e_{2}, x\right)+\left|\Psi^{\prime}\left(e_{2}\right)\right|^{r} L_{9}\left(e_{1}, e_{2}, x\right)- \\
-\mu \zeta^{\sigma}\left(e_{2}, e_{1}\right) P_{3}\left(e_{1}, e_{2}, x\right)
\end{array}\right]^{\frac{1}{r}}} \\
+\left[L_{10}\left(e_{1}, e_{2}, x\right)\right]^{\frac{1}{p}}\left[\begin{array}{c}
\left|\Psi^{\prime}\left(e_{1}\right)\right|^{r} L_{11}\left(e_{1}, e_{2}, x\right)+\left|\Psi^{\prime}\left(e_{2}\right)\right|^{r} L_{12}\left(e_{1}, e_{2}, x\right)- \\
-\mu \zeta^{\sigma}\left(e_{2}, e_{1}\right) P_{4}\left(e_{1}, e_{2}, x\right)
\end{array}\right]^{\frac{1}{r}}
\end{array}\right]
$$

where

$$
\begin{aligned}
L_{7}\left(e_{1}, e_{2}, x\right) & :=\int_{0}^{\wp_{\zeta}(x)} \ell^{p} d \ell=\frac{1}{p+1}\left[\wp_{\zeta}(x)\right]^{p+1}, \\
L_{8}\left(e_{1}, e_{2}, x\right) & :=\int_{0}^{\wp_{\zeta}(x)}(1-\ell) d \ell=\wp_{\zeta}(x)-\frac{1}{2}\left[\wp_{\zeta}(x)\right]^{2}, \\
L_{9}\left(e_{1}, e_{2}, x\right) & :=\int_{0}^{\wp_{\zeta}(x)} \ell d \ell=\frac{1}{2}\left[\wp_{\zeta}(x)\right]^{2}, \\
L_{10}\left(e_{1}, e_{2}, x\right):= & \int_{\wp_{\zeta}(x)}^{1}(1-\ell)^{p} d \ell=\frac{1}{p+1}\left(\frac{e_{1}+\zeta\left(e_{2}, e_{1}\right)-x}{\zeta\left(e_{2}, e_{1}\right)}\right)^{p+1}, \\
L_{11}\left(e_{1}, e_{2}, x\right):= & \int_{\wp_{\zeta}(x)}^{1}(1-\ell) d \ell=\frac{e_{1}+\zeta\left(e_{2}, e_{1}\right)-x}{\zeta\left(e_{2}, e_{1}\right)}-\frac{1}{2}\left(1-\left[\wp_{\zeta}(x)\right]^{2}\right), \\
L_{12}\left(e_{1}, e_{2}, x\right) & :=\int_{\wp_{\zeta}(x)}^{1} \ell d \ell=\frac{1}{2}\left(1-\left[\wp_{\zeta}(x)\right]^{2}\right),
\end{aligned}
$$

and

$$
\begin{aligned}
& P_{3}\left(e_{1}, e_{2}, x\right):=\int_{0}^{\wp_{\zeta}(x)} \ell(1-\ell) d \ell=\frac{1}{2}\left[\wp_{\zeta}(x)\right]^{2}-\frac{1}{3}\left[\wp_{\zeta}(x)\right]^{3}, \\
& P_{4}\left(e_{1}, e_{2}, x\right):=\int_{\wp_{\zeta}(x)}^{1} \ell(1-\ell) d \ell=L_{12}\left(e_{1}, e_{2}, x\right)-\frac{1}{3}\left(1-\left[\wp_{\zeta}(x)\right]^{3}\right) .
\end{aligned}
$$

II. Taking $\mathrm{q} \rightarrow 1^{-}, \mu \rightarrow 0^{+}$and $x=\frac{2 e_{1}+\zeta\left(e_{2}, e_{1}\right)}{2}$ in Theorem 1, we get [16, Theorem 6].

$$
\begin{aligned}
& \left|\Psi\left(\frac{2 e_{1}+\zeta\left(e_{2}, e_{1}\right)}{\zeta\left(e_{2}, e_{1}\right)}\right)-\frac{1}{\zeta\left(e_{2}, e_{1}\right)} \int_{e_{1}}^{e_{1}+\zeta\left(e_{2}, e_{1}\right)} \Psi(\ell) d \ell\right| \\
& \leq \frac{\zeta\left(e_{2}, e_{1}\right)}{16}\left(\frac{4}{p+1}\right)^{\frac{1}{p}}\left[\left(3\left|\Psi^{\prime}\left(e_{1}\right)\right|^{r}+\left|\Psi^{\prime}\left(e_{2}\right)\right|^{r}\right)^{\frac{1}{r}}+\left(\left|\Psi^{\prime}\left(e_{1}\right)\right|^{r}+3\left|\Psi^{\prime}\left(e_{2}\right)\right|^{r}\right)^{\frac{1}{r}}\right] .
\end{aligned}
$$


III. Taking $x=\frac{q e_{1}+e_{2}}{1+\mathrm{q}}, \mu \rightarrow 0^{+}$and $\zeta\left(e_{2}, e_{1}\right)=e_{2}-e_{1}$ in Theorem 1, we obtain [1, Theorem 18].

$$
\begin{aligned}
& \left|\Psi\left(\frac{q e_{1}+e_{2}}{1+\mathrm{q}}\right)-\frac{1}{e_{2}-e_{1}} \int_{e_{1}}^{e_{2}} \Psi(\ell)_{e_{1}} d_{\mathrm{q}} \ell\right| \leq \\
& \mathrm{q}\left(e_{2}-e_{1}\right)\left[\left(\frac{1}{(1+\mathrm{q})^{p+1}} \frac{(1-\mathrm{q})}{1-\mathrm{q}^{p+1}}\right)\left[\frac{\left.\left.\left(\mathrm{q}^{2}+2 \mathrm{q}\right)\right|_{e_{1}} D_{\mathrm{q}} \Psi\left(e_{1}\right)\right|^{r}}{(1+\mathrm{q})^{3}}+\frac{\mathrm{q}^{2}\left|e_{e_{1}} D_{\mathrm{q}} \Psi\left(e_{2}\right)\right|^{r}}{(1+\mathrm{q})^{3}}\right]^{\frac{1}{r}}+\right. \\
& \left.\left(\int_{\frac{1}{1+\mathrm{q}}}^{1}\left(\ell-\frac{1}{\mathrm{q}}\right)^{p} e_{1} d_{\mathrm{q}} \ell\right)^{\frac{1}{p}}\left[\frac{\left.\left.\left(\mathrm{q}^{3}+\mathrm{q}^{2}-\mathrm{q}\right)\right|_{e_{1}} D_{\mathrm{q}} \Psi\left(e_{1}\right)\right|^{r}}{(1+\mathrm{q})^{3}}+\frac{\left.\left.\left(\mathrm{q}^{2}+2 \mathrm{q}\right)\right|_{e_{1}} D_{\mathrm{q}} \Psi\left(e_{2}\right)\right|^{r}}{(1+\mathrm{q})^{3}}\right]^{\frac{1}{r}}\right],
\end{aligned}
$$

where

$$
\int_{\frac{1}{1+\mathrm{q}}}^{1}\left(\ell-\frac{1}{\mathrm{q}}\right)^{p}{ }_{0} d_{\mathrm{q}} \ell=(1-\mathrm{q})\left[\begin{array}{c}
\sum_{n=0}^{\infty} \mathrm{q}^{n}\left(\mathrm{q}^{n}-\frac{1}{\mathrm{q}}\right)^{p} \\
-\frac{1}{1+\mathrm{q}} \sum_{n=0}^{\infty} \mathrm{q}^{n}\left(\mathrm{q}^{n}\left(\frac{1}{1+\mathrm{q}}\right)-\frac{1}{\mathrm{q}}\right)^{p}
\end{array}\right] .
$$

IV. Taking $\mu \rightarrow 0^{+}$and $x=\frac{e_{1}+\mathrm{q}\left(e_{1}+\zeta\left(e_{2}, e_{1}\right)\right)}{1+\mathrm{q}}$ in Theorem 1, we get

$$
\begin{aligned}
& \left|\Psi\left(\frac{e_{1}+\mathrm{q}\left(e_{1}+\zeta\left(e_{2}, e_{1}\right)\right)}{1+\mathrm{q}}\right)-\frac{1}{\zeta\left(e_{2}, e_{1}\right)} \int_{e_{1}}^{e_{1}+\zeta\left(e_{2}, e_{1}\right)} \Psi(\ell)_{e_{1}} d_{\mathrm{q}} \ell\right| \leq \mathrm{q} \zeta\left(e_{2}, e_{1}\right) \\
& \times\left\{\left(\frac{\mathrm{q}^{p}}{(1+\mathrm{q})^{p+1}} \frac{(1-\mathrm{q})}{1-\mathrm{q}^{p+1}}\right)\left[\frac{\left.\left.\left(\mathrm{q}^{3}+\mathrm{q}^{2}+\mathrm{q}\right)\right|_{e_{1}} D_{\mathrm{q}} \Psi\left(e_{1}\right)\right|^{r}}{(1+\mathrm{q})^{3}}+\frac{\left.\left.\mathrm{q}^{2}\right|_{\left.e_{1} D_{\mathrm{q}} \Psi\left(e_{2}\right)\right|^{r}}\right]^{\frac{1}{r}}}{(1+\mathrm{q})^{3}}\right]\right. \\
& \left.+\left(\int_{\frac{\mathrm{q}}{1+q}}^{1}\left(\ell-\frac{1}{\mathrm{q}}\right)^{p}{ }_{0} d_{\mathrm{q}} \ell\right)^{\frac{1}{p}}\left[\frac{\left.\left.\mathrm{q}^{2}\right|_{e_{1}} D_{\mathrm{q}} \Psi\left(e_{1}\right)\right|^{r}}{(1+\mathrm{q})^{3}}+\frac{\left.\left.(1+2 \mathrm{q})\right|_{e_{1}} D_{\mathrm{q}} \Psi\left(e_{2}\right)\right|^{r}}{(1+\mathrm{q})^{3}}\right]^{\frac{1}{r}}\right\},
\end{aligned}
$$

where

$$
\int_{\frac{\mathrm{q}}{1+\mathrm{q}}}^{1}\left(\ell-\frac{1}{\mathrm{q}}\right)^{p}{ }_{0} d_{\mathrm{q}} \ell=(1-\mathrm{q})\left[\begin{array}{c}
\sum_{n=0}^{\infty} \mathrm{q}^{n}\left(\mathrm{q}^{n}-\frac{1}{\mathrm{q}}\right)^{p} \\
-\frac{\mathrm{q}}{1+\mathrm{q}} \sum_{n=0}^{\infty} \mathrm{q}^{n}\left(\mathrm{q}^{n}\left(\frac{\mathrm{q}}{1+\mathrm{q}}\right)-\frac{1}{\mathrm{q}}\right)^{p}
\end{array}\right] .
$$

Theorem 2. Let $\Psi: P \rightarrow \mathbb{R}$ be a function such that ${ }_{e_{1}} D_{\mathrm{q}} \Psi$ is q-integrable on $P^{\circ}$. If $\left.\left.\right|_{e_{1}} D_{\mathrm{q}} \Psi\right|^{r}$ is strongly preinvex functions of order $\sigma>0$ with modulus $\mu>0$ on $P$, then for $r \geq 1$, the following inequality holds:

$$
\left|\Psi(x)-\frac{1}{\zeta\left(e_{2}, e_{1}\right)} \int_{e_{1}}^{e_{1}+\zeta\left(e_{2}, e_{1}\right)} \Psi(\ell)_{e_{1}} d_{\mathrm{q}} \ell\right| \leq \zeta\left(e_{2}, e_{1}\right)
$$




$$
\times\left[\begin{array}{c}
{\left[J_{1}\left(\mathrm{q}, e_{1}, e_{2}, x\right)\right]^{1-\frac{1}{r}}\left[\begin{array}{c}
\left|e_{1} D_{\mathrm{q}} \Psi\left(e_{1}\right)\right|^{r} J_{2}\left(\mathrm{q}, e_{1}, e_{2}, x\right) \\
+\left|e_{1} D_{\mathrm{q}} \Psi\left(e_{2}\right)\right|^{r} J_{3}\left(\mathrm{q}, e_{1}, e_{2}, x\right)- \\
-\mu \zeta^{\sigma}\left(e_{2}, e_{1}\right) Q_{1}\left(\mathrm{q}, e_{1}, e_{2}, x\right)
\end{array}\right]^{\frac{1}{r}}} \\
+\left[J_{4}\left(\mathrm{q}, e_{1}, e_{2}, x\right)\right]^{1-\frac{1}{r}}\left[\begin{array}{c}
\left|e_{1} D_{\mathrm{q}} \Psi\left(e_{1}\right)\right|^{r} J_{5}\left(\mathrm{q}, e_{1}, e_{2}, x\right) \\
+\left|e_{1} D_{\mathrm{q}} \Psi\left(e_{2}\right)\right|^{r} J_{6}\left(\mathrm{q}, e_{1}, e_{2}, x\right)- \\
-\mu \zeta^{\sigma}\left(e_{2}, e_{1}\right) Q_{2}\left(\mathrm{q}, e_{1}, e_{2}, x\right)
\end{array}\right]^{\frac{1}{r}}
\end{array}\right],
$$

where

$$
\begin{aligned}
J_{1}\left(\mathrm{q}, e_{1}, e_{2}, x\right) & :=\int_{0}^{\wp_{\zeta}(x)} \mathrm{q} \ell_{0} d_{\mathrm{q}} \ell=\frac{\mathrm{q}}{1+\mathrm{q}}\left[\wp_{\zeta}(x)\right]^{2}, \\
J_{2}\left(\mathrm{q}, e_{1}, e_{2}, x\right):= & \int_{0}^{\wp_{\zeta}(x)}\left(\mathrm{q} \ell-\mathrm{q} \ell^{2}\right)_{0} d_{\mathrm{q}} \ell=J_{1}\left(\mathrm{q}, e_{1}, e_{2}, x\right)-J_{3}\left(\mathrm{q}, e_{1}, e_{2}, x\right), \\
J_{3}\left(\mathrm{q}, e_{1}, e_{2}, x\right): & =\int_{0}^{\wp_{\zeta}(x)} \mathrm{q} \ell^{2}{ }_{0} d_{\mathrm{q}} \ell=\frac{\mathrm{q}}{1+\mathrm{q}+\mathrm{q}^{2}}\left[\wp_{\zeta}(x)\right]^{3}, \\
J_{4}\left(\mathrm{q}, e_{1}, e_{2}, x\right): & =\int_{\wp_{\zeta}(x)}^{1}(\mathrm{q} \ell-1){ }_{0} d_{\mathrm{q}} \ell=\frac{\mathrm{q}}{1+\mathrm{q}}\left(\frac{e_{1}+\zeta\left(e_{2}, e_{1}\right)-x}{\zeta\left(e_{2}, e_{1}\right)}\right)^{2}, \\
J_{5}\left(\mathrm{q}, e_{1}, e_{2}, x\right): & =\int_{\wp_{\zeta}(x)}^{1}\left(1-\mathrm{q} \ell-\ell+\mathrm{q} \ell^{2}\right){ }_{0} d_{\mathrm{q}} \ell=J_{4}\left(\mathrm{q}, e_{1}, e_{2}, x\right)-J_{6}\left(\mathrm{q}, e_{1}, e_{2}, x\right), \\
J_{6}\left(\mathrm{q}, e_{1}, e_{2}, x\right) & :=\int_{\wp_{\zeta}(x)}^{1}\left(\mathrm{q} \ell^{2}-\ell\right)_{0} d_{\mathrm{q}} \ell= \\
& =\frac{1}{(1+\mathrm{q})\left(1+\mathrm{q}+\mathrm{q}^{2}\right)}-\frac{1}{1+\mathrm{q}}\left[\wp_{\zeta}(x)\right]^{2}+\frac{\mathrm{q}}{1+\mathrm{q}+\mathrm{q}^{2}}\left[\wp_{\zeta}(x)\right]^{3},
\end{aligned}
$$

and

$$
\begin{aligned}
Q_{1}\left(\mathrm{q}, e_{1}, e_{2}, x\right) & :=\int_{0}^{\wp_{\zeta}(x)} \mathrm{q} \ell^{2}(1-\ell)_{0} d_{\mathrm{q}} \ell= \\
& =\frac{\mathrm{q}}{1+\mathrm{q}+\mathrm{q}^{2}}\left[\wp_{\zeta}(x)\right]^{3}-\frac{\mathrm{q}}{1+\mathrm{q}+\mathrm{q}^{2}+\mathrm{q}^{3}}\left[\wp_{\zeta}(x)\right]^{4}, \\
Q_{2}\left(\mathrm{q}, e_{1}, e_{2}, x\right) & :=\int_{\wp_{\zeta}(x)}^{1} \ell(\mathrm{q} \ell-1)(1-\ell)_{0} d_{\mathrm{q}} \ell=-\frac{1}{1+\mathrm{q}}\left(1-\left[\wp_{\zeta}(x)\right]^{2}\right)+ \\
+\frac{(1+\mathrm{q})}{1+\mathrm{q}+\mathrm{q}^{2}} & \left(1-\left[\wp_{\zeta}(x)\right]^{3}\right)-\frac{\mathrm{q}}{1+\mathrm{q}+\mathrm{q}^{2}+\mathrm{q}^{3}}\left(1-\left[\wp_{\zeta}(x)\right]^{4}\right) .
\end{aligned}
$$


Proof. Using Lemma 1 strongly preinvexity of order $\sigma>0$ with modulus $\mu>0$ of $\left|e_{1} D_{\mathrm{q}} \Psi\right|^{r}$ and the well known power mean inequality, we have

$$
\begin{aligned}
& \left|\Psi(x)-\frac{1}{\zeta\left(e_{2}, e_{1}\right)} \int_{e_{1}}^{e_{1}+\zeta\left(e_{2}, e_{1}\right)} \Psi(\ell)_{e_{1}} d_{\mathrm{q}} \ell\right| \\
& \leq \zeta\left(e_{2}, e_{1}\right)\left[\begin{array}{c}
\left.\left.\int_{0}^{\wp_{\zeta}(x)} \mathrm{q} \ell\right|_{e_{1}} D_{\mathrm{q}} \Psi\left(e_{1}+\ell \zeta\left(e_{2}, e_{1}\right)\right)\right|_{0} d_{\mathrm{q}} \ell \\
+\left.\left.\int_{\wp_{\zeta}(x)}^{1}(\mathrm{q} \ell-1)\right|_{e_{1}} D_{\mathrm{q}} \Psi\left(e_{1}+\ell \zeta\left(e_{2}, e_{1}\right)\right)\right|_{0} d_{\mathrm{q}} \ell
\end{array}\right] \\
& \leq \zeta\left(e_{2}, e_{1}\right)\left[\left(\int_{0}^{\wp_{\zeta}(x)} \mathrm{q} \ell_{0} d_{\mathrm{q}} \ell\right)^{1-\frac{1}{r}}\left(\left.\left.\int_{0}^{\wp_{\zeta}(x)} \mathrm{q} \ell\right|_{e_{1}} D_{\mathrm{q}} \Psi\left(e_{1}+\ell \zeta\left(e_{2}, e_{1}\right)\right)\right|^{r}{ }_{0} d_{\mathrm{q}} \ell\right)^{\frac{1}{r}}\right. \\
& \left.+\left(\int_{\wp_{\zeta}(x)}^{1}(\mathrm{q} \ell-1)_{0} d_{\mathrm{q}} \ell\right)^{1-\frac{1}{r}}\left(\left.\left.\int_{\wp_{\zeta}(x)}^{1}(\mathrm{q} \ell-1)\right|_{e_{1}} D_{\mathrm{q}} \Psi\left(e_{1}+\ell \zeta\left(e_{2}, e_{1}\right)\right)\right|^{r}{ }_{0} d_{\mathrm{q}} \ell\right)^{\frac{1}{r}}\right] \\
& \leq \zeta\left(e_{2}, e_{1}\right)\left\{\left(\int_{0}^{\wp_{\zeta}(x)} \mathrm{q} \ell_{0} d_{\mathrm{q}} \ell\right)^{1-\frac{1}{r}}\right. \\
& \times\left(\left|e_{1} D_{\mathrm{q}} \Psi\left(e_{1}\right)\right|^{r} \int_{0}^{\wp_{\zeta}(x)} \mathrm{q} \ell(1-\ell)_{0} d_{\mathrm{q}} \ell+\left|{ }_{e_{1}} D_{\mathrm{q}} \Psi\left(e_{2}\right)\right|^{r} \int_{0}^{\wp_{\zeta}(x)} \mathrm{q} \ell^{2}{ }_{0} d_{\mathrm{q}} \ell-\mu \zeta^{\sigma}\left(e_{2}, e_{1}\right)\right. \\
& \left.\int_{0}^{\wp_{\zeta}(x)} \mathrm{q} \ell^{2}(1-\ell)_{0} d_{\mathrm{q}} \ell\right)^{\frac{1}{r}}+\left(\int_{\wp_{\zeta}(x)}^{1}(\mathrm{q} \ell-1)_{0} d_{\mathrm{q}} \ell\right)^{1-\frac{1}{r}} \\
& \times\left[\left|e_{1} D_{\mathrm{q}} \Psi\left(e_{1}\right)\right|^{r} \int_{\wp_{\zeta}(x)}^{1}(\mathrm{q} \ell-1)(1-\ell)_{0} d_{\mathrm{q}} \ell+\left.\left.\right|_{e_{1}} D_{\mathrm{q}} \Psi\left(e_{2}\right)\right|^{r} \int_{\gamma_{\zeta}(x)}^{1}(\mathrm{q} \ell-1) \ell_{0} d_{\mathrm{q}} \ell\right. \\
& \left.\left.-\mu \zeta^{\sigma}\left(e_{2}, e_{1}\right) \int_{\wp_{\zeta}(x)}^{1} \ell(\mathrm{q} \ell-1)(1-\ell)_{0} d_{\mathrm{q}} \ell\right]^{\frac{1}{r}}\right\} .
\end{aligned}
$$

The proof of Theorem 2 is completed.

We point out some special cases of Theorem 2 . 
Corollary 2.1. I. Taking $r=1$ in Theorem 2, we have

$$
\begin{aligned}
& \left|\Psi(x)-\frac{1}{\zeta\left(e_{2}, e_{1}\right)} \int_{e_{1}}^{e_{1}+\zeta\left(e_{2}, e_{1}\right)} \Psi(\ell)_{e_{1}} d_{\mathrm{q}} \ell\right| \leq \zeta\left(e_{2}, e_{1}\right) \times \\
& {\left[\begin{array}{c}
\left|e_{1} D_{\mathrm{q}} \Psi\left(e_{1}\right)\right|\left[J_{2}\left(\mathrm{q}, e_{1}, e_{2}, x\right)+J_{5}\left(\mathrm{q}, e_{1}, e_{2}, x\right)\right]+ \\
+\left|e_{1} D_{\mathrm{q}} \Psi\left(e_{2}\right)\right|\left[J_{3}\left(\mathrm{q}, e_{1}, e_{2}, x\right)+J_{6}\left(\mathrm{q}, e_{1}, e_{2}, x\right)\right]- \\
-\mu \zeta^{\sigma}\left(e_{2}, e_{1}\right)\left(Q_{1}\left(\mathrm{q}, e_{1}, e_{2}, x\right)+Q_{2}\left(\mathrm{q}, e_{1}, e_{2}, x\right)\right)
\end{array}\right] .}
\end{aligned}
$$

II. Taking $r=1$ and $\mathrm{q} \rightarrow 1^{-}$in Theorem 2, we get

$$
\begin{aligned}
& \left|\Psi(x)-\frac{1}{\zeta\left(e_{2}, e_{1}\right)} \int_{e_{1}}^{e_{1}+\zeta\left(e_{2}, e_{1}\right)} \Psi(\ell) d \ell\right| \leq \zeta\left(e_{2}, e_{1}\right) \times \\
& {\left[\begin{array}{c}
\left|\Psi^{\prime}\left(e_{1}\right)\right|\left[J_{7}\left(e_{1}, e_{2}, x\right)+J_{9}\left(e_{1}, e_{2}, x\right)\right]+ \\
+\left|\Psi^{\prime}\left(e_{2}\right)\right|\left[J_{8}\left(e_{1}, e_{2}, x\right)+J_{10}\left(e_{1}, e_{2}, x\right)\right]- \\
-\mu \zeta^{\sigma}\left(e_{2}, e_{1}\right)\left(Q_{3}\left(e_{1}, e_{2}, x\right)+Q_{4}\left(e_{1}, e_{2}, x\right)\right)
\end{array}\right],}
\end{aligned}
$$

where

$$
\begin{aligned}
& J_{7}\left(e_{1}, e_{2}, x\right):=\int_{0}^{\wp_{\zeta}(x)} \ell(1-\ell) d \ell=\frac{1}{2}\left[\wp_{\zeta}(x)\right]^{2}-\frac{1}{3}\left[\wp_{\zeta}(x)\right]^{3}, \\
& J_{8}\left(e_{1}, e_{2}, x\right):=\int_{0}^{\wp_{\zeta}(x)} \ell^{2} d \ell=\frac{1}{3}\left[\wp_{\zeta}(x)\right]^{3}, \\
& J_{9}\left(e_{1}, e_{2}, x\right):= \int_{\wp_{\zeta}(x)}^{1}\left(1-2 \ell+\ell^{2}\right) d \ell=\frac{1}{3}-\wp_{\zeta}(x)+\left[\wp_{\zeta}(x)\right]^{2}-\frac{1}{3}\left[\wp_{\zeta}(x)\right]^{3}, \\
& J_{10}\left(e_{1}, e_{2}, x\right):=\int_{\wp_{\zeta}(x)}^{1}\left(\ell-\ell^{2}\right) d \ell=\frac{1}{6}-\frac{1}{2}\left[\wp_{\zeta}(x)\right]^{2}+\frac{1}{3}\left[\wp_{\zeta}(x)\right]^{3},
\end{aligned}
$$

and

$$
\begin{aligned}
& Q_{3}\left(e_{1}, e_{2}, x\right):=\int_{0}^{\wp_{\zeta}(x)} \ell^{2}(1-\ell) d \ell=\frac{1}{3}\left[\wp_{\zeta}(x)\right]^{3}-\frac{1}{4}\left[\wp_{\zeta}(x)\right]^{4}, \\
& Q_{4}\left(e_{1}, e_{2}, x\right):=\int_{\wp_{\zeta}(x)}^{1} \ell(\ell-1)(1-\ell) d \ell \\
& =-\frac{1}{2}\left(1-\left[\wp_{\zeta}(x)\right]^{2}\right)+\frac{2}{3}\left(1-\left[\wp_{\zeta}(x)\right]^{3}\right)-\frac{1}{4}\left(1-\left[\wp_{\zeta}(x)\right]^{4}\right) .
\end{aligned}
$$


III. Taking $\mathrm{q} \rightarrow 1^{-}, \mu \rightarrow 0^{+}$and $x=\frac{2 e_{1}+\zeta\left(e_{2}, e_{1}\right)}{2}$ in Theorem 2, we obtain [16, Theorem 8].

$$
\begin{aligned}
& \left|\Psi\left(\frac{2 e_{1}+\zeta\left(e_{2}, e_{1}\right)}{\zeta\left(e_{2}, e_{1}\right)}\right)-\frac{1}{\zeta\left(e_{2}, e_{1}\right)} \int_{e_{1}}^{e_{1}+\zeta\left(e_{2}, e_{1}\right)} \Psi(\ell) d \ell\right| \\
& \leq \frac{\zeta\left(e_{2}, e_{1}\right)}{8}\left[\left(\frac{2\left|\Psi^{\prime}\left(e_{1}\right)\right|^{r}+\left|\Psi^{\prime}\left(e_{2}\right)\right|^{r}}{3}\right)^{\frac{1}{r}}+\left(\frac{\left|\Psi^{\prime}\left(e_{1}\right)\right|^{r}+2\left|\Psi^{\prime}\left(e_{2}\right)\right|^{r}}{3}\right)^{\frac{1}{r}}\right] .
\end{aligned}
$$

IV. Taking $r=1, \mathrm{q} \rightarrow 1^{-}, \mu \rightarrow 0^{+}$and $x=\frac{2 e_{1}+\zeta\left(e_{2}, e_{1}\right)}{2}$ in Theorem 2, we get [16, Theorem 5].

$$
\left|\Psi\left(\frac{2 e_{1}+\zeta\left(e_{2}, e_{1}\right)}{\zeta\left(e_{2}, e_{1}\right)}\right)-\frac{1}{\zeta\left(e_{2}, e_{1}\right)} \int_{e_{1}}^{e_{1}+\zeta\left(e_{2}, e_{1}\right)} \Psi(\ell) d \ell\right| \leq \frac{\zeta\left(e_{2}, e_{1}\right)}{8}\left[\left|\Psi^{\prime}\left(e_{1}\right)\right|+\left|\Psi^{\prime}\left(e_{2}\right)\right|\right] .
$$

IV. Taking $\mu \rightarrow 0^{+}$and $x=\frac{q e_{1}+e_{2}}{1+q}$ and $\zeta\left(e_{2}, e_{1}\right)=e_{2}-e_{1}$ in Theorem 2, we have the following inequalities, for more details, see [7].

$$
\begin{aligned}
& \left|\Psi\left(\frac{q e_{1}+e_{2}}{1+\mathrm{q}}\right)-\frac{1}{e_{2}-e_{1}} \int_{e_{1}}^{e_{2}} \Psi(\ell)_{e_{1}} d_{\mathrm{q}} \ell\right| \leq\left(e_{2}-e_{1}\right) \times \\
& {\left[\begin{array}{l}
\frac{1}{(1+\mathrm{q})^{3-\frac{3}{r}}}\left[\left|e_{1} D_{\mathrm{q}} \Psi\left(e_{1}\right)\right|^{r} \frac{\mathrm{q}^{2}(1+\mathrm{q})}{\left(1+\mathrm{q}+\mathrm{q}^{2}\right)(1+\mathrm{q})^{3}}+\left.\left.\right|_{e_{1}} D_{\mathrm{q}} \Psi\left(e_{2}\right)\right|^{r} \frac{\mathrm{q}}{\left(1+\mathrm{q}+\mathrm{q}^{2}\right)(1+\mathrm{q})^{3}}\right]^{\frac{1}{r}}+ \\
\left(\frac{\mathrm{q}}{1+\mathrm{q}}\right)^{3-\frac{3}{r}}\left[\left.\left.\right|_{e_{1}} D_{\mathrm{q}} \Psi\left(e_{1}\right)\right|^{r} \frac{\left(\mathrm{q}^{5}+\mathrm{q}^{4}+\mathrm{q}^{3}-2 \mathrm{q}\right)}{\left(1+\mathrm{q}+\mathrm{q}^{2}\right)(1+\mathrm{q})^{3}}+\left.\left.\right|_{e_{1}} D_{\mathrm{q}} \Psi\left(e_{2}\right)\right|^{r} \frac{2 \mathrm{q}}{\left(1+\mathrm{q}+\mathrm{q}^{2}\right)(1+\mathrm{q})^{3}}\right]^{\frac{1}{r}}
\end{array}\right] .}
\end{aligned}
$$

V. Taking $r=1, \mu \rightarrow 0^{+}, x=\frac{q e_{1}+e_{2}}{1+q}$ and $\zeta\left(e_{2}, e_{1}\right)=e_{2}-e_{1}$ in Theorem 2, we obtain [1, Theorem 13].

$$
\begin{aligned}
& \left|\Psi\left(\frac{q e_{1}+e_{2}}{1+\mathrm{q}}\right)-\frac{1}{e_{2}-e_{1}} \int_{e_{1}}^{e_{2}} \Psi(\ell)_{e_{1}} d_{\mathrm{q}} \ell\right| \leq\left(e_{2}-e_{1}\right) \times \\
& {\left[\left.\right|_{e_{1}} D_{\mathrm{q}} \Psi\left(e_{1}\right)\left|\frac{\left(\mathrm{q}^{5}+\mathrm{q}^{4}+2 \mathrm{q}^{3}+\mathrm{q}^{2}-2 \mathrm{q}\right)}{\left(1+\mathrm{q}+\mathrm{q}^{2}\right)(1+\mathrm{q})^{3}}+\right| e_{e_{1}} D_{\mathrm{q}} \Psi\left(e_{2}\right) \mid \frac{3 \mathrm{q}}{\left(1+\mathrm{q}+\mathrm{q}^{2}\right)(1+\mathrm{q})^{3}}\right] .}
\end{aligned}
$$

VI. Taking $\mu \rightarrow 0^{+}$and $x=\frac{e_{1}+\mathrm{q}\left(e_{1}+\zeta\left(e_{2}, e_{1}\right)\right)}{1+\mathrm{q}}$ in Theorem 2, we get

$$
\begin{aligned}
& \left|\Psi\left(\frac{e_{1}+\mathrm{q}\left(e_{1}+\zeta\left(e_{2}, e_{1}\right)\right)}{1+\mathrm{q}}\right)-\frac{1}{\zeta\left(e_{2}, e_{1}\right)} \int_{e_{1}}^{e_{1}+\zeta\left(e_{2}, e_{1}\right)} \Psi(\ell)_{e_{1}} d_{\mathrm{q}} \ell\right| \leq \zeta\left(e_{2}, e_{1}\right) \times \\
& {\left[\begin{array}{l}
\left(\frac{\mathrm{q}}{1+\mathrm{q}}\right)^{3-\frac{3}{r}}\left[\left.\left.\right|_{e_{1}} D_{\mathrm{q}} \Psi\left(e_{1}\right)\right|^{r} \frac{\mathrm{q}^{3}\left(1+\mathrm{q}^{2}\right)}{\left(1+\mathrm{q}+\mathrm{q}^{2}\right)(1+\mathrm{q})^{3}}+\left.\left.\right|_{e_{1}} D_{\mathrm{q}} \Psi\left(e_{2}\right)\right|^{r} \frac{\mathrm{q}^{4}}{\left(1+\mathrm{q}+\mathrm{q}^{2}\right)(1+\mathrm{q})^{3}}\right]^{\frac{1}{r}}+ \\
\left(\frac{\mathrm{q}}{(1+\mathrm{q})^{3}}\right)^{1-\frac{1}{r}}\left[\left|e_{1} D_{\mathrm{q}} \Psi\left(e_{1}\right)\right|^{r} \frac{\left(2 \mathrm{q}^{3}+\mathrm{q}^{2}-\mathrm{q}-1\right)}{\left(1+\mathrm{q}+\mathrm{q}^{2}\right)(1+\mathrm{q})^{3}}+\left.\left.\right|_{e_{1}} D_{\mathrm{q}} \Psi\left(e_{2}\right)\right|^{r} \frac{\left(1+2 \mathrm{q}-\mathrm{q}^{3}\right)}{\left(1+\mathrm{q}+\mathrm{q}^{2}\right)(1+\mathrm{q})^{3}}\right]^{\frac{1}{r}}
\end{array}\right] .}
\end{aligned}
$$




\section{Conclusion}

It is expected that from the results obtained, and following the methodology applied, additional special functions may also be evaluated. Future works can be developed in the area of numerical analysis and even contributions using quantum algorithms, using the theorems and corollaries presented. Finally, our results can be applied to derive some inequalities using special means and error estimations. The authors hope that the ideas and techniques of this paper will inspire interested readers working in this fascinating field.

Acknowledgements. The authors would like to thank the honorable referees and editors for valuable comments and suggestions for improved our manuscript.

\section{REFERENCES}

[1] N. Alp, M.Z. Sarikaya, M. Kunt, Í. Íşcan, q-Hermite Hadamard inequalities and quantum estimates for midpoint type inequalities via convex and quasi-convex functions, Journal of King Saud University-Science, 30(2) (2018), 193-203.

[2] M.U. Awan, M.A. Noor, F. Safdar, K.I. Noor, Higher order strongly preinvex functions and inequalities, Preprint, (2019).

[3] Y. Deng, M.U. Awan, S. Wu, Quantum integral inequalities of Simpson-type for strongly preinvex functions, , Mathematics, 7 (2019), pp. 14.

[4] A.B. Israel, B. Mond, What is invexity? J. Austral. Math. Soc. Ser. B, 28 (1986), 1-9.

[5] V. Kac, P. Cheung, Quantum calculus, Universitext, Springer, New York, 2002, pp. 113.

[6] S. Karamardian, The nonlinear complementarity problem with applications, Part 2, J. Optim. Theory Appl., 4 (1969), 167-181.

[7] M. Kunt, A. Kashuri, T.S. Du, Quantum Montgomery identity and some quantum integral inequalities, arXiv:1907.03601, (2019), pp. 16.

[8] G.H. Lin, M. Fukushima, Some exact penalty results for nonlinear programs and mathematical programs with equilibrium constraints, J. Optim. Theory Appl., 118 (2003), 67-80.

[9] D.S. Mitrinović, J.E. Pečarić, A.M. Fink, Inequalities involving functions and their integrals and derivatives, Kluwer Academic, Dordrecht, 1991, pp. 602.

[10] B.B. Mohsin, M.U. Awan, M.A. Noor, L. Riahi, K.I. Noor, B. Almutairi, New quantum Hermite-Hadamard inequalities utilizing harmonic convexity of the functions, IEEE Access, 7 (2019), 20479-20483.

[11] M.A. Noor, G. Cristescu, M.U. Awan, Bounds having Riemann type quantum integrals via strongly convex functions, Studia Sci. Math. Hung., 54(2) (2017), 221-240.

[12] M.A. Noor, K.I. Noor, M.U. Awan, Some quantum integral inequalities via preinvex functions, Appl. Math. Comput. 269 (2015), 242-251.

[13] M.A. Noor, K.I. Noor, M.U. Awan, Some quantum estimates for Hermite-Hadamard inequalities, Appl. Math. Comput. 251 (2015), 675-679.

[14] M.A. Noor, K.I. Noor, M.U. Awan, J. Li, On Hermite-Hadamard Inequalities for h-preinvex functions, Filomat, 28(7) (2014), 1463-1474.

[15] B.T. Polyak, Existence theorems and convergence of minimizing sequences in extremum problems with restrictions, Sov. Math. Dokl., 7 (1966), 72-75.

[16] M.Z. Sarikaya, H. Bozkurt, N. Alp, On Hermite-Hadamard type integral inequalities for preinvex and log-preinvex functions, (2012), pp. 13.

[17] W. Sudsutad, S.K. Ntouyas, J. Tariboon, J., Quantum integral inequalities for convex functions, J. Math. Inequal., 9(3) (2015), 781-793. 
[18] J. Tariboon, S.K. Ntouyas, Quantum integral inequalities on finite intervals, J. Inequal. Appl., 2014, Article ID 121 (2014).

[19] M.J. Vivas-Cortez, R. Liko, A. Kashuri, J.E.H. Hernández, New quantum estimates of trapezium-type inequalities for generalized $\phi$-convex functions, Mathematics, 7(11) (2019), pp. 19.

[20] M.J. Vivas-Cortez, A. Kashuri, R. Liko, J.E.H. Hernández, Quantum estimates of Ostrowski inequalities for generalized $\phi$-convex functions, Symmetry, 11(12) (2019), pp. 16.

[21] M.J. Vivas-Cortez, A. Kashuri, R. Liko, J.E.H. Hernández, Some inequalities using generalized convex functions in quantum analysis, Symmetry, 11(11) (2019), pp. 14.

[22] T. Weir, B. Mond, Preinvex functions in multiple objective optimization, J. Math. Anal. Appl., 136(1) (1988), 29-38.

[23] Y. Zhang, T.S. Du, H. Wang, Y.J. Shen, Different types of quantum integral inequalities via ( $\alpha, m)$-convexity, J. Inequal. Appl., 2018(264) (2018), pp. 24.

1 Department of Mathematics,

Faculty of Technical Science,

University "Ismail Qemali",

9400 Vlora, Albania

Email address: artionkashuri@gmail.com

Email address: rozanaliko86@gmail.com

2 Department of Mathematics and Computer Science,

Alabama State University,

Montgomery, AL 36101, USA

Email address: enwaeze@alasu.edu

Received 03.04.2021

Revised 06.12.2021

Accepted 10.01.2022 